\title{
Zur Theorie des Elektrons II
}

\author{
Von Walter Wessel \\ USAF Institute of Technology, Wright Field, Dayton, Ohio (USA) \\ (Z. Naturforschg. 6 a, 478-483 [1951]; eingegangen am 27. April 1951)
}

\begin{abstract}
Das im ersten Teil dieser Arbeit aufgestellte Programm für die Bestimmung des Massenoperators wird mit einigen Einschränkungen zu Ende geführt. Die auf klassischem Wege dafür gefundene Form steht im Einklange mit einer inzwischen aufgefundenen, quantentheoretischen Bedingung. Wesentlich für die Durchführung ist eine von zehn neuen Identitäten der D i r a c schen Algebra.
\end{abstract}

$\mathrm{D}$ vorliegende Theorie sucht, wie schon einige Male auseinandergesetzt wurde, das Auftreten der neuen Variablen, mit denen der Elektronenspin in Diracs Theorie beschrieben wird, aus dem Auftreten höherer Zeitableitungen in der klassischen Bewegungsgleichung des Elektrons zu erklären und damit D i r a c s Theorie zugleich zu verallgemeinern. In dem vorangehenden Teile dieser Arbeit ${ }^{1}$ hatte das zur Aufstellung eines geschlossenen Systems („Ringes“) von P o is s on - Klammern geführt, die einerseits die klassischen Bewegungsgleichungen möglichst getreu aus einer $\mathrm{Hamilt}$ an schen Funktion ableiten, anderseits durch Umdeutung in Vertauschungsrelationen zur Quantelung führen sollten. Von diesen Programmpunkten ist inzwischen der zweite durch Arbeiten von $\mathrm{B}$ o p p und $\mathrm{B}$ a u e ${ }^{2}$ und dem Verfasser ${ }^{3}$ weitgehend erledigt worden, und es sind auch neue Gesichtspunkte für den ersten aufgetreten. Wir kommen damit auf die Frage der Bewegungsgleichungen zurück. Wie in ThE I sind die folgenden Betrachtungen, von einigen quantentheoretischen Ausblicken abgesehen, rein klassischer Art.

Die Bewegungsgleichungen sind insofern noch unbestimmt, als die Ruhmasse in einer willkürlichen Art von den Invarianten der Spinmechanik abhängen kann. Der Grund für diese Willkür ist durch B o p p ${ }^{4}$ sehr klar geworden: er entspricht, wenigstens in dem $\mathrm{Maße}$, wie sich diese Theorie aus einem Variationsprinzip ableiten läßt, der Unbestimmtheit der Dichtefunktion bzw. der „cut-off“-Funktion, die die endliche Ausdehnung des Elektrons beschreibt. B o p p und andere Autoren (vgl. ${ }^{4}$ ) haben schon eine Reihe von Funktionen dafür angesetzt, ohne eine davon

1 W. W e s s e 1, Z. Naturforschg. 1, 622 [1946], abgedruckt in FIAT-Report 1131. Im folgenden als ThE I zitiert.

2 F. B op p u. F. L. B a u e r, Z. Naturforschg. 4 a, 611 [1949]; F. L. B a u e r, ebenda 4a, 720 [1949]. überzeugend ausgezeichnet zu finden. Die Bestimmung des Massenoperators ist damit zu einem Hauptproblem der hier versuchten Theorie geworden.

Man braucht zu seiner Lösung nicht unbedingt von einer Dichtefunktion auszugehen, deren Existenz sowieso etwas zweifelhaft ist. Wir haben im allgemeinen (für $I \neq 0$, vgl. die Diskussion in QM I) gar kein Hamiltonsches Prinzip, sondern nur eine Hamiltonsche Funktion. Es genügt, wenn der Massenoperator als solcher wohldefiniert ist, und hierzu wird man auf quantenth e or e t i s c h e m Wege zu einem stark einschränkenden Kriterium geführt, wenn man von den unendlichen Darstellungen des Verfassers ${ }^{3}$ ausgeht. Man erhält dann im allgemeinen eine Häufung der Masseneigenwerte gegen die Null und entgeht dem nur, mindestens im Sinne einer notwendigen Bedingung ${ }^{5}$, wenn der Massenoperator als Funktion einer der erwähnten Invarianten entweder nichtintegrable Singularitäten im Endlichen aufweist oder im Unendlichen wesentlich - wie ein cosinus hyperbolicus - singulär wird. Alle bisher versuchten Funktionen werden damit ausgeschlossen.

Es fragt sich, was dieses Verhalten, das zu einer recht unbequemen, singulären Integralgleichung für die Massen führt, klassisch zu bedeuten hat, und ob man vielleicht durch klassische Argumente diese Funktion vollständiger bestimmen kann. In der Tat war in ThE I eine darauf bezügliche Fragestellung unerledigt geblieben. Wir hatten ursprünglich versucht, die Reaktionskraft der Strahlung in ihrer vollen, nichtkonservativen Form in die Bewegungsgleichungen aufzunehmen. Die weitere Entwicklung hatte das als undurchführbar erwiesen und schließlich in ThE I durch Gesichtspunkte der Kompatibilität zur

3 W. W e s s e l, Physic. Rev. 76, 1512 [1949]; Z. Naturforschg. 4a, 645 [1949]. Im folgenden als QM I zitiert.

4 F. B o p p, Z. Naturforschg. 3a, 564 [1948].

5 W. W e s s e l, Z. Naturforschg. 6a, 473 [1951]. 
Ableitung der Bewegungsgleichungen aus einer $\mathrm{H}$ amilton-Funktion geführt, die in der Neubegründung durch $\mathrm{B}$ o $\mathrm{p} \mathrm{p}^{4}$ darauf hinauskommt, daß man nur den reversiblen Teil der Strahlungskraft (bis zu einer höheren Näherung) berücksichtigt. Sobald man nun in dieser Hamilton-Funktion überhaupt eine Abhängigkeit des Massenoperators von den Invarianten der Spinmechanik annimmt, ist die Bewegung des Ladungsschwerpunktes immer ungleichförmig beschleunigt, auch bei reiner Trägheitsbewegung (konstantem Impuls). Daher besteht auch immer eine irreversible Ausstrahlung, im letztgenannten Falle das korrespondenzmäßige Gegenstück zu dem Vermö gien des Diracschen Elektrons, auch bei kräftefreier Bewegung gegebenenfalls in ein "Loch“ zu springen. Der Gedanke, die dabei ausgestrahlte Energie ein$f_{a c h}$ zu ignorieren, erscheint doch wohl nur überzeugend, wenn damit die Hamilton-Funktion völlig bestimmt wird.

In der Quantenelektrodynamik wird die Konstanz der Energie dadurch gewahrt, daß man den ausgestrahlten Teil in der Feldenergie mitzählt. Diese erscheint additiv neben dem Massenoperator ${ }^{6}$; die neuere Entwicklung ist geradezu gekennzeichnet durch die Schwierigkeit, sie reinlich davon zu trennen. Wenn man das bedenkt, kommt man fast zwangsläufig zu einem Prinzip, auf das wir schon vor längerer Zeit ${ }^{7}$ durch Gesichtspunkte formaler Einfachheit geführt wurden und das in ThE I unerfüllt blieb: man berücksichtige auch in der „Feldmechanik“ den irreversiblen Teil der Strahlungskraft dadurch, daß man die ausgestrahlte Energie in der Ruhmasse mitzählt. Wenn das möglich ist, und man wird sehen, daß es in gewissen Grenzen gelingt, hat man eine physikalische Vorschrift zur Festlegung des Massenoperators, denn dieser ist ja die einzige noch verbleibende Unbekannte in den Bewegungsgleichungen.

Der Grund, weshalb wir in ThE I mit diesem Prinzip nicht durchkamen, war neben der damals noch bestehenden Unsicherheit in der Deutung der ganzen Spinmechanik besonders der Umstand, daß die Konstanten der inneren Bewegung im Rahmen der klassischen Theorie als nicht bestimmbar erschienen. Inzwischen hat die quantentheoretische Analyse nicht nur diese Konstanten deuten helfen und ihre Eigen-

6 Vgl. etwa E. F e rmi, Rev. mod. Physics 4, 131 [1932] oder W. Heitler, The Quantum Theorie of Radiation (Oxford 1936) Chap. III, $\$ 10$.

7 W. W e s s e l, Naturwiss. 30, 606 [1942]; Ann. Physik (5) 43, 565 [1943]. werte festgelegt, sondern überdies zu der Erkenntnis geführt, daß ein bestimmtes Zwischenintegral der Bewegungsgleichungen zu den Identitäten der zugrundeliegenden Algebra gehört und daher gar nicht frei wählbar ist. Damit wird die obengenannte Vorschrift wohldefiniert, wenn man sich auf die kräftefreie Bewegung beschränkt und annimmt, daß sich das Teilchen mit einer bestimmten Masse im Zustande seines kleinsten Spins befindet. Sie ist leicht ausführbar und liefert für den Massenoperator tatsächlich eine Funktion mit dem auf Grund der quantentheoretischen Untersuchung zu verlangenden Verhalten, zufällig gerade in der einfachsten Form eines hyperbolischen Cosinus für alle Argumentwerte. Die Beschränkung auf den kleinsten Spin ist wesentlich, weil nur dieser eine L o r e n t z - Invariante ist. Äußere Felder müßten vielleicht noch im Massenoperator berücksichtigt werden. Jedenfalls wird der Operator der unbeschleunigten Ruhmasse in seiner analytischen Form mit Einschluß einer Fundamentalkonstanten (der Feinstrukturkonstanten) auf diese Weise völlig festgelegt.

1. Die Identitäten der Spinmechanik

Die von uns benutzten Variablen sind nach dem Muster der Diracschen Feldgrößen aufgebaut und unterliegen in Folge davon einer Reihe von Identitäten. Die Fülle dieser Beziehungen ist durch die Beiträge mehrerer Autoren, auf die sich der erste Teil dieser Arbeit stützte, noch nicht ausgeschöpft. Wir wollen auch hier, um die Spinoralgebra nicht wieder aufrollen zu müssen, von einer Systematik absehen und nur die wirklich gebrauchten Beziehungen noch einmal übersichtlich und ergänzt zusammenstellen.

Natürlich sind diese Identitäten partikuläre Integrale der Bewegungsgleichungen. Man muß sich fragen, ob sie wesentlich sind, da nur mit ihrer Hilfe das Problem völlig bestimmt wird. Wir wollen uns hier auf den Standpunkt stellen, daß diejenigen Identitäten wesentlich sind, die auch aus dem Variationsprinzip bei B o p p folgen. Nicht alle daraus folgenden sind wesentlich (vgl. QM I), doch sind umgekehrt alle hier benutzten auch von Bopps Variablen erfüllt.

Das Teilchen wird also beschrieben ${ }^{8}$, außer durch Koordinaten und Impulse, durch seine vom Impuls unabhängige Vierergeschwindigkeit $u_{k}$ und den Tensor $M_{i k}, i, k=1 \ldots 4$, seines magnetischen und elektrischen Momentes; von dem sich übrigens das elektrische nachher auch noch als abhängig erweisen wird. Wir schreiben auch $M$ und $\Pi$ für die Vektoren mit 
den Komponenten $M_{23}, M_{31}, M_{12}$ bzw. $M_{14}, M_{24}$, $M_{34}$. Der Spin ist dann durch ${ }^{9}$

$$
\sigma(\sigma+1)=M^{2}
$$

gegeben, da $-M \hbar$ in Verbindung mit dem Bahndrehimpuls in Zentralfeldern konstant ist [vgl. ThE I, Gl. (6. 22) bzw. (7.22) oder B op p ${ }^{10}$ ]. Quantentheoretisch sind die Werte von $\sigma$ wegen der Unendlichkeit der Matrizen nach oben nicht beschränkt, d.h. der Spin ist wie ein gewöhnlicher Drehimpuls „angeregter" Werte fähig. Es gibt aber einen tiefsten Wert dafür, und dieser ist durch die Invarianten des vollen Momententensors festgelegt. Wir schreiben dazu

$$
\begin{aligned}
M^{2}-\Pi^{2} & =I^{2}-K^{2} \\
M I I & =I K
\end{aligned}
$$

bzw. $I^{2}-K^{2}-1$, wenn es sich um die Operatoren handelt. Die Forderung der Hermitezität führt dann dazu [vgl. QM I (1.3)], daß $\sigma$ nicht kleiner als $|I|$ sein kann. Mit Hinblick auf dieses quantentheoretische Ergebnis sei es erlaubt, auch in dieser klassischen Theorie $I$ als den „kleinsten Spin“ zu bezeichnen.

Außer in der wohlbekannten Bedeutung von (1.1) tritt nun der Spin in dieser relativistischen Theorie noch in einer sehr ähnlichen Verbindung mit den vierten Komponenten der Vierergeschwindigkeit und ihrer Zugeordneten auf, deren Natur bisher nicht erkannt wurde. Die Vierergeschwindigkeit erscheint bei uns als zeitartiger Einheitsvektor in Richtung eines Vektors $\iota_{k}, k=1 \ldots 4$, mit der Invarianten

$$
\iota_{k} \iota^{k}=-\left(I^{2}+K^{2}\right)
$$

(bzw. $I^{2}+K^{2}-1$ ) als

$$
u_{k}=\iota_{k} / \sqrt{I^{2}+K^{2}} .
$$

8 Die in ThE I verwendeten $j_{i}, k_{i}, M_{i k}, I, J$ waren dimensioniert. Wir führen sie hier auf dimensionslose $\iota_{i}, \varkappa_{i}, M_{i k}, I, K$ zurück, indem wir die Skalare und Vektoren in Vielfachen von $\Gamma \hbar$, die Momente in Vielfachen von $\Gamma \Lambda \hbar$ ausdrücken. Es soll also z. B. $i_{k}=\Gamma \hbar \iota_{k}$ sein. Damit fallen $\Gamma$ und $A$ heraus, und die Poisson-Klammern - vgl. z. B. (2.7) weiter unten - nehmen einen Faktor $1 / \hbar$ an. Sie werden dann bei der Verwandlung in Vertauschungsrelationen von $\hbar$ frei, während umgekehrt in den Bewegungsgleichungen (2.3)-(2.6) ein Faktor $\hbar$ stehen bleibt. Bei B o p p kommt dieser Faktor herein durch Entwicklung nach der elementaren Länge $l$. Es ist merkwürdig und entspricht einer vom Verfasser oft hervorgehobenen Schwierigkeit in der Interpretation des Spins durch Strahlungskräfte, daß man $l$ von der Größenordnung der Compton-Wellenlänge und nicht etwa des Elektronenradius anzunehmen hat (vgl. B o p p ${ }^{4}$; die Annahme $\varrho=1$ führt auf $\left.l=2 \hbar / m_{0} c\right)$.
Mit $\iota^{k}$ und $M_{i k}$ läßt sich ein raumartiger Vektor $\varkappa_{i}$ bilden, der dadurch gekennzeichnet ist, daß er parallel $\iota^{k} M_{k i}$ und vom Betrage

$$
\varkappa_{k} x^{k}=I^{2}+K^{2}
$$

ist. Er bestimmt eine „zugeordnete“ Geschwindigkeit (raumartiger Einheitsvektor)

$$
U_{k}=x_{k} / \sqrt{I^{2}+K^{2}} .
$$

Die drei Größen $\iota^{4}, \varkappa^{4}$ und $K$ bilden nun einen Unterring von $\mathrm{P}$ o is s o $\mathrm{n}$ - Klammern bzw. Vertauschungsrelationen, was dazu führt ${ }^{11}$, daß sie sämtlich mit

$$
Q^{2}=\left(\varkappa^{4}\right)^{2}+K^{2}-\left(\iota^{4}\right)^{2}
$$

vertauschbar sind. Diese Beziehung trat zunächst als partikuläres Integral auf. Es erschienen positive und negative Werte von $\varrho^{2}$ als zulässig, wobei die positiven eine einfache korrespondenzmäßige Deutung verschiedener Mesonenmassen als möglich erscheinen ließen ${ }^{11}$. Diese Möglichkeit bestand jedoch, wie wir schon a. a. O. betonten, in den vorhandenen quantentheoretischen Darstellungen nicht, denn diese gründeten sich auf

$$
\varrho^{2}=-\sigma(\sigma+1)=-M^{2} .
$$

In der Tat gehört die Beziehung

$$
\left(\varkappa^{4}\right)^{2}+K^{2}-\left(\iota^{4}\right)^{2}+M^{2}=0
$$

zu den Identitäten der Diracschen Algebra. Das erscheint zunächst verwunderlich, da sie ja keine Invariante ist. Sie bildet vielmehr in Verbindung mit (1.2) die 44-Komponente eines symmetrischen Tensors,

$$
\varkappa_{i} \varkappa^{k}+M_{i l} M^{k l}-\iota_{i} \iota^{k}-I^{2} \delta_{i}^{k}=0,
$$

der natürlich nur als Ganzes verschwinden kann. In den Spinoren von ThE I geschrieben bedeutet das

$$
k_{\varrho \sigma} k^{\dot{\mu} v}+\frac{1}{\Lambda^{2}} m_{\varrho}^{\dot{\mu}} m_{\sigma}^{v}-i_{\varrho \sigma} j^{\dot{\mu} v}+\delta_{\varrho}^{\dot{\mu}} \delta_{\sigma}^{v} \Delta \bar{\Delta} \equiv 0 .
$$

Tatsächlich werden diese Beziehungen durch die Größen (3.4), (3.7) bzw. (4.4), (4.7) a. a. O. identisch

9 In QM I wurde $\sigma$ durch $\varkappa$ bezeichnet, was sich aber in Verbindung mit dem Vierervektor $\varkappa^{k}$ als unpraktisch erwiesen hat.

10 F. B o p p, Z. Naturforschg. 1, 196 [1946]. Man beachte jedoch, daß unser $M$ nicht als Vektorprodukt darstellbar und daher $\sigma$ quantenmechanisch nicht auf ganzzahlige Werte beschränkt ist.

${ }_{11}$ W. We s s e l, Z. Naturforschg. 3a, 559 [1948]. 
erfüllt. Sie gelten auch bei B o p p und B a u er, wo sie wegen des Verschwindens von $I$ und wegen der von 1 bis 5 laufenden Summation einfach

$$
g_{\gamma^{a} a} g_{\gamma \beta}-g_{\alpha} g_{\beta} \equiv 0
$$

lauten, wie die Autoren selbst bemerken. Mit Hinblick darauf werden wir diese zehn Identitäten, insbesondere (1.10), als wesentlich betrachten.

Die Wahl der unabhängigen Veränderlichen ist danach auf viele Weisen möglich. Ihre Anzahl beträgt 7 , wie schon $\mathrm{K}$ of in $\mathrm{k}^{12}$ ausgerechnet hat. In der Tat bauen sich die 16 Variablen auf 4 komplexen Spinoren auf, von denen, wie ebenfalls B opp und $\mathrm{B}$ a u e r bemerkten, noch eine Phase willkürlich bleibt; die Vektoren und Tensoren von ThE I sind nämlich invariant gegen eine Transformation

$$
\psi \rightarrow \psi e^{i \varphi}, \chi \rightarrow \chi e^{-i \varphi}
$$

der Spinoren. Die natürlichste Wahl vom physikalischen Standpunkte ist wohl, die drei Komponenten der Geschwindigkeit mit dem Vektor des magnetischen Momentes und seinem Minimalwert $I$ als Unabhängige zu betrachten. Auf die verschiedenen Möglichkeiten der Elimination brauchen wir nicht einzugehen; es sei nur zur Berichtigung eines Versehens in QM I bemerkt, daß die Invarianten $j^{i} M_{i k}$ und $j^{i} M_{i}^{*} k$ nicht von den andern Beziehungen unabhängig sind, sondern zusammen die Relation (1.2) ergeben.

\section{Die Differentialgleichung des Massenoperators}

Die Bewegungsgleichungen übernehmen wir unverändert aus ThE I. Ein Strich bedeutet die Ableitung nach der Weltlinie. $m$ ist die Masse, die von $I$ und $K$ abhängen kann. Da $I$ zeitkonstant (und diskret gequantelt) ist, ist die wesentliche Unabhängige $K$. Diese Größe ist auch quantentheoretisch kontinuierlich veränderlich. $m(I, K)$ bestimmt die Energie durch

$$
u_{k} g^{k}=-m(I, K) c .
$$

$g^{k}, k=1 \ldots 4$, ist der Impuls mit Einschluß des Viererpotentials. Durch Übergang von $u_{k}$ zu $U_{k}$ entsteht eine „zugeordnete“ Masse:

$$
U_{k} g^{k}=-M c,
$$

deren Mitführung, ebenso wie die von $U_{k}$, formale Erleichterungen bietet.

12 W. K o f i n k, Ann. Physik (5) 38, 421 [1940].
In der neuen Schreibweise ${ }^{8}$ lauten die Bewegungsgleichungen (bezüglich des Auftretens der

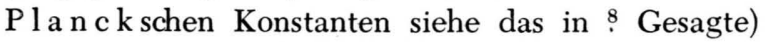

$u_{i}^{\prime}=\frac{K}{\hbar\left(I^{2}+K^{2}\right)}\left\{m c U_{i}-M c u_{i}-g^{l} M_{l i} / K\right\}+\frac{1}{\hbar} U_{i} \frac{\partial m c}{\partial K}$,

$$
\begin{gathered}
U_{i}^{\prime}=\frac{K}{\pi\left(I^{2}+K^{2}\right)}\left\{m c u_{i}-M c U_{i}-g_{i}\right\}+\frac{1}{\hbar} u_{i} \frac{\partial m c}{\partial \ell} \\
K^{\prime}=\frac{1}{\hbar} M c, \quad I^{\prime}=0 \\
\cdot M_{i k}^{\prime}=\frac{1}{\hbar}\left(u_{i} g_{k}-u_{k} g_{i}\right) .
\end{gathered}
$$

Die P o is s o n - Klammern nehmen mit ${ }^{8}$ die Form an

$$
\left(M_{1}, M_{2}\right)=\frac{1}{\hbar} M_{3}
$$

usw., vgl. QM I (3.1). Durch Ersetzen der runden Klammern durch das Vertauschungssymbol und Einführung eines Faktors $i \hbar$ rechterhand entstehen daraus die Vertauschungsrelationen, wie sie den Darstellungen ${ }^{3}$ zugrunde liegen.

Unser Prinzip verlangt nun [vgl. ThE I (7.2) bzw. (8.2)] eine Zunahme der Masse gemäß

$$
m^{\prime}=\frac{2}{3} \frac{e^{2}}{c^{2}} u_{i}^{\prime} u^{i^{\prime}} .
$$

Die linke Seite dieser Gleichung lautet mit (2.5)

$$
m^{\prime}=\frac{\partial m}{\partial K} K^{\prime}=\frac{1}{\hbar} \frac{\partial m}{\partial K} \dot{M} c .
$$

Rechterhand in (2.8) entstehen durch Quadrieren von (2.3) zwei quadratische Invarianten und ein gemischtes Glied. Wir betrachten zunächst dieses, d. h. den Faktor von $\frac{\partial m c}{\partial K}$. Er lautet bis auf weitere Faktoren

$$
m c U_{i} U^{i}-M c u_{i} U^{i}-g^{i} M_{j i} U^{i} / K .
$$

Hier ist $U_{i} U^{i}=1, u_{i} U^{i}=0$, letzteres weil $U^{i}$ parallel $u_{j} M^{j i}$ ist. Ferner gilt nach ThE I (5.2) bzw. (6.2) in der Schreibweise ${ }^{8}$

$$
M_{i i} U^{i}=-M_{i j} U^{i}=-K u_{i} .
$$

Das letzte Glied in (2.10) wird daher $\mathrm{zu}^{j} u_{j}$, und das ist nach (2.1) gleich $-m c$. Damit verschwindet das gemischte Produkt (2.10).

Die quadratischen Invarianten lassen sich ganz ähnlich ausrechnen. Für $g^{j} M_{j i} u^{i}$ gilt (2.11) mit vertauschten $U^{i}$ und $u^{i}$. Schließlich bleibt 


$$
\begin{aligned}
u_{i}^{\prime} u^{i^{\prime}}=\frac{K^{2}}{i^{2}\left(I^{2}+K^{2}\right)^{2}}\left\{(M c)^{2}-(m c)^{2}\right. \\
\left.\quad+g^{l} M_{l i} g_{k} M^{k i} / K\right\}+\frac{1}{\hbar^{2}}\left(\frac{\partial m c}{\partial K}\right)^{2} .
\end{aligned}
$$

Durch Einsetzen der rechten Seiten von (2.9) und (2.12) in (2.8) ergibt sich eine Differentialgleichung für $m(I, K)$. Sie ist freilich in dieser Form nicht lösbar, weil bei weitem mehr als nur $I$ und $K$ darin eingehen.

\section{Integration im Schwerpunkts-} s y s te m

Wir setzen nach dem in der Einleitung Gesagten alle äußeren Kräfte gleich Null und gehen zum Schwerpunktssystem über: $g^{k}=0, k=1,2,3$, $g^{4}=p^{4}=m_{0} c$, wobei $m_{0}$ die Ruhmasse im Sinne eines bestimmten Observablenwertes ist. Aus (2.1) ergibt sich dann mit Hinblick auf (1.5):

$$
m=m_{0} \iota^{4} / \sqrt{I^{2}+K^{2}}
$$

(beachte $\iota_{4}=-\iota^{4}$ ) und ebenso aus (2.2) mit Hinblick auf (1.7):

$$
M=m_{0} \varkappa^{4} / \sqrt{I^{2}+K^{2}},
$$

sowie schließlich

$$
g^{l} M_{l i} g_{k} M^{k i}=g^{4} M_{4 i} g_{4} M^{4 i}=\left(m_{0} c\right)^{2} I I^{2} .
$$

Mit alledem wird der Klammerausdruck in (2.12) bis auf einen Faktor $\left(m_{0} c\right)^{2}$ :

$$
\frac{\left(\varkappa^{4}\right)^{2}-\left(\iota^{4}\right)^{2}}{I^{2}+K^{2}}+\frac{\Pi^{2}}{K^{2}} \text {. }
$$

Wir machen nun von den Identitäten (1.2) und (1.10) Gebrauch. Mit ihrer Hilfe läßt sich der Klammerausdruck (3.4) auf die Form bringen

$$
\frac{I^{2}}{K^{2}} \frac{M^{2}-I^{2}}{I^{2}+K^{2}} \text {. }
$$

Er verschwindet für $I=0$, bleibt aber für höhere Werte des Minimalspins bestehen und ist dann von $M^{2}$ abhängig, was keine Invariante ist. Wir werden aber auch davon frei, wenn wir die genaue Gültigkeit unseres Prinzips auf den Fall einschränken, daß die Partikel sich im Zustande ihres niedrigsten Spins

$$
M=|I|
$$

befindet. Dann verschwindet (3.5) auch für $I \neq 0$, und wir haben nach (2.12) einfach

$$
u_{i}^{\prime} u^{i^{\prime}}=\frac{1}{\hbar^{2}}\left(\frac{\partial m c}{\partial K}\right)^{2} .
$$

In dieser Gestalt gilt die Formel auch bei B op p, sogar allgemein für nicht-verschwindende Felder. Der Grund dafür ist das identische Verschwinden von $I$. Damit werden die Geschwindigkeitskomponenten vertauschbar [vgl. unsere Gln. (5.11) bzw. (6.11) in ThE I], was dazu führt, daß die Beschleunigung von den Impulsen und damit auch von den Potentialen unabhängig wird. Der Massenterm ist dann der einzige Term in der Hamilton-Funktion, mit dem die Geschwindigkeitskomponenten nicht vertauschbar sind.

Das Quadrat der Viererbeschleunigung und damit nach der Lorentzschen Formel die Ausstrahlung sind also auch in diesem aufs Äußerste vereinfachten Falle nicht gleich Null, solange $m$ von $K$ irgendwie abhängt, und unser Prinzip (2.8) führt zu einer nichttrivialen Bedingung. Einsetzen von (2.9) und (3.7) ergibt

$$
\frac{2}{3} \frac{e^{2}}{\hbar c} \frac{\partial m}{\partial K}=M
$$

Wir haben nun nach (3.2) und (3.1), wenn wir wieder (1.10) und (3.6) benutzen,

$$
\begin{aligned}
M^{2}-m^{2}=m_{0}{ }^{2} & \frac{\left(x^{4}\right)^{2}-\left(\iota^{4}\right)^{2}}{I^{2}+K^{2}} \\
& =-m_{0}{ }^{2} \frac{M^{2}+K^{2}}{I^{2}+K^{2}}=-m_{0}{ }^{2},
\end{aligned}
$$

also nach (3.8)

$$
\frac{\partial m}{\partial K}=\frac{3}{2} \frac{\hbar c}{e^{2}} \sqrt{m^{2}-m_{0}^{2}},
$$

und daraus ergibt sich durch Integration die einfache Formel

$$
m(I, K)=m_{0} \mathcal{E}_{0}\left\{\left(\frac{3 \hbar c}{2 e^{2}} K+\text { const. }\right) .\right.
$$

Hier ist, wie gesagt, $m_{0}$ zunächst ein bestimmter Observablenwert. Für einen solchen Wert läßt sich also tatsächlich alles erfüllen. Es liegt nun nahe, $m_{0}$ in (3.11) als universell bzw. nur noch von $I$ abhängig zu betrachten und eventuell weitere, durch $g^{4} / c$ in (2.1) definierte Werte der Ruhmasse als Eigenwerte der entsprechenden Matrix zuzulassen, wobei man eine Durchbrechung der Bedingung (2.8) ebenso in Kauf zu nehmen hätte wie für höhere Spinwerte. Das Ergebnis (3.11) entspricht dann ganz den quantentheoretischen Erfordernissen, insofern als es auf eine der einfachst möglichen Weisen die in ${ }^{5}$ aufgefundene Bedingung erfüllt. 
4. Diskussion der D if ferenzen-

$$
\text { gleichung }
$$

Für die mathematische Auswertung von (3.11) steht zunächst die Integralgleichung a.a.O. ${ }^{5}$ (2.5) zur Verfügung. Sie ließe sich allenfalls auch streng fassen, ist aber mit (3.11) schon in ihrer unstrengen Form numerisch sehr schwer diskutierbar, zumal sich die Integrationskonstante in (3.11) nicht festlegen läßt. Der naheliegende Versuch, sie Null zu setzen und den reziproken Massenoperator damit $\mathrm{zu}$ bestimmen, daß man $1 / \mathfrak{S o j} \frac{3 \hbar c}{2 e^{2}} K$ wie eine $\delta$-Funktion behandelt, führt leider auf eine Matrix mit einer (schwach) divergenten Säkulargleichung. Die genauere Behandlung wird dann gleich unüberwindlich schwierig. Die Verhältnisse werden aber sehr übersichtlich, wenn man mit (3.11) in die in ${ }^{5}$ abgeleitete Differenzengleichung (4.16) eingeht. .Diese lautet dann, wenn man zur Abkürzung

und

$$
m / m_{0}=\mu
$$

$$
\frac{3}{2} \frac{\hbar c}{e^{2}}=\Gamma
$$

setzt und zur vorläufigen Orientierung die Konstante

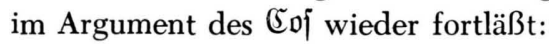

$$
\begin{aligned}
\frac{\mu}{2}\left\{\left(\frac{1}{2}-i K\right) \varphi_{m}(\right. & \left.K-i)+\left(\frac{1}{2}+i K\right) \varphi_{m}(K+i)\right\} \\
= & \operatorname{Sof} \Gamma K\left(\frac{1}{4}+K^{2}\right)^{1 / 2} \varphi_{m}(K) .
\end{aligned}
$$

Man erkennt wohl ziemlich leicht, welcher Funktionstyp wesentlich in dieser Gleichung steckt: es ist die Funktion

$$
\varphi_{m}=e^{ \pm i \frac{\Gamma}{2} K^{2}} \text {. }
$$

Die linke Seite von (4.3) geht damit über in

$\frac{\mu}{2}\left\{\left(\frac{1}{2}-i K\right) e^{ \pm \Gamma K}+\left(\frac{1}{2}+i K\right) e^{\mp \Gamma K}\right\} \varphi_{m} e^{\mp i \Gamma / 2}$,

und man hätte in (4.4) eine genaue Eigenfunktion von (4.3) zum Eigenwerte $\mu=1$, wenn die rechte Seite von (4.3)

$$
\left(\frac{1}{2} \operatorname{Sof} \Gamma K \mp i K \operatorname{Sin} \Gamma K\right) e^{\mp i \Gamma / 2}
$$

hieße. Der Absolutbetrag hiervon fällt sowohl für kleine wie besonders für große $K$ sehr schnell mit der rechten Seite von (4.3) zusammen, und eine Linearisierung der Quadratwurzel in (4.3) im Sinne von (4.6) liegt in der Linie einer quantenmechanischen Umdeutung.

Wir möchten $\mathrm{zu}$ einer solchen Umdeutung im Rahmen dieser Untersuchung noch keinen Ansatz machen, denn man muß sich dabei vor allem fragen, wie die Antipartikel in diese unendlichen Darstellungen eingeführt werden soll. Hierzu muß natürlich die vollständige Wellengleichung mit Einschluß der Impuls- und Potentialglieder herangezogen werden, und die ist bei uns nicht so einfach zu diskutieren wie bei Dirac, weil die Geschwindigkeitskomponenten bzw. die $\iota^{k}, k=1,2,3$, kontinuierliche Eigenwerte haben und daher ihre Matrizen, auch wenn man sie nach der Drehungsgruppe ausreduziert, immer noch eine Matrix mit Streckenspektrum als Faktor enthalten. Mit dem Vorliegenden sollte nur gezeigt werden, daß die klassische Theorie dem quantentheoretischen Bedürfnis nach einem singulären Massenoperator durchaus entgegenkommt und daß sich die Dinge auch mathematisch in eine einfache Form bringen lassen. 